\title{
Identification of a novel variant erythrocyte surface antigen-1 (VESA1) in Babesia orientalis
}

\author{
Zhen $\mathrm{Han}^{1,2} \cdot$ Zheng $\mathrm{Ni}^{1,2} \cdot$ Xiang Shu ${ }^{1,2} \cdot$ Yaxin Zheng ${ }^{1,2} \cdot$ Wanxin Luo ${ }^{1,2} \cdot$ Hongyan Zhang $^{1,2} \cdot$ Yingjun Xia $^{1,2}$. \\ Fangjie $\mathrm{Li}^{1,2} \cdot \operatorname{Lan} \mathrm{He}^{1,2,3}$. Junlong $\mathrm{Zhao}^{1,2,3}$
}

Received: 22 December 2020 / Accepted: 24 May 2021 / Published online: 5 July 2021

(c) The Author(s), under exclusive licence to Springer-Verlag GmbH Germany, part of Springer Nature 2021

\begin{abstract}
Babesia orientalis, belonging to the phylum Apicomplexa, is mainly accountable for water buffalo babesiosis, which adversely affected the livestock industry in China. Variant erythrocyte surface antigen-1 (VESA1), an antigen that helps infected erythrocytes to escape from host immune responses, was first reported in Babesia bovis. Various VESA1 proteins have also been characterized in other Babesia species. Nevertheless, there is no research on the identification and characterization of VESA1 proteins in Babesia orientalis. In this study, the BoVESA1 gene was amplified from both gDNA and cDNA. The results revealed that it is an intronless gene with a full length of $753 \mathrm{bp}$, encoding a protein of 250 amino acids with a predicted molecular weight of $28 \mathrm{kDa}$. The coding sequence (CDS) was cloned into the pGEX-6p-1 vector using a homologous recombination kit and expressed as a glutathione-S-transferase (GST)-fusion protein with a molecular weight of $53 \mathrm{kDa}$. The tertiary structure of BoVESA1 was predicted using the I-TASSER software. The recombinant protein was subjected to western blotting; the immunogenicity of recombinant BoVESA1 (rBoVESA1) was identified by incubating it with $B$. orientalis-positive serum. The native BoVESA1 was identified using the lysates of $B$. orientalis-infected water buffalo erythrocytes incubated with the anti-rBoVESA1 mouse serum. The results showed a band of $\sim 28 \mathrm{kDa}$, which is similar to the predicted size. Immunofluorescence assay (IFA) using anti-rBoVESA1 serum probed indicated a strong signal in the infected RBCs, while the negative control showed no signal. In conclusion, the VESA1 protein was first identified in $B$. orientalis. This study facilitated further investigation of $B$. orientalis, and the results indicated that BoVESA1 may serve as a potential candidate antigen for diagnosis and detection of $B$. orientalis infection.
\end{abstract}

Keywords Antigenic variation $\cdot$ Babesiosis $\cdot$ Babesia orientalis $\cdot$ Immunogenicity $\cdot$ BoVESA1

Section Editor: Dana Mordue

Junlong Zhao

zhaojunlong@mail.hzau.edu.cn

Zhen Han

841659862@qq.com

Zheng Nie

785648484@qq.com

Xiang Shu

739669325@qq.com

Yaxin Zheng

775368143@qq.com

Wanxin Luo

2847909660@qq.com

Hongyan Zhang

1396945739@qq.com

Yingjun Xia

1424584845@qq.com
Fangjie Li

1147228199@qq.com

Lan He

helan@mail.hzau.edu.cn

1 State Key Laboratory of Agricultural Microbiology, College of Veterinary Medicine, Huazhong Agricultural University, Wuhan 430070, Hubei, China

2 Key Laboratory of Preventive Veterinary Medicine in Hubei Province, The Cooperative Innovation Center for Sustainable Pig Production, Wuhan 430070, Hubei, China

3 Key Laboratory of Development of Veterinary Diagnostic Products, Ministry of Agriculture of the People's Republic of China, Wuhan 430070, Hubei, China 


\section{Introduction}

Babesia spp. taxonomically belongs to the parasitic protozoa of the phylum Apicomplexa (He et al. 2017). It can infect a variety of animals, including humans and mice, with severe acute disease characterized by sequestration of mature parasites; the attributes of the disease are high fever, hemoglobinuria, and a respiratory distress syndrome-like condition (Novacco et al. 2019; Wright et al. 1988). Ticks including Boophilus microplus, Boophilus annulatus, Ixodes persulcatus, and Rhipicephalus haemaphysaloides are main vectors of Babesia spp. (Klompen et al. 1996; Young and Morzaria 1986). Generally, after a larva or nymph feeds on the blood of infected animals, Babesia spp. can be transmitted by nymphs or adult ticks during their next stage of development (Berdyev 1985). Hemoparasites pose a serious threat to the livestock population worldwide, mostly affecting livestock industry in subtropical and tropical regions throughout the world. Babesia orientalis, a Babesia species, has been identified in water buffalo in southern and central China, which lead to a massive economic loss to the Chinese buffalo industry (Kivaria 2006; Ristic 1988).

Microbes such as Plasmodium falciparum, African trypanosomes, Candida albicans, and Borrelia hermsii establish long-term existence in host cells via antigenic variation mechanism which is a rapid alteration in the structure and antigenicity of essential components (Arnarson et al. 2017; Biswal et al. 2019; Deitsch et al. 1997; Langsley and Roth 1987; Palmer et al. 2016). The pathogen-synthesized components which appeared during the course of a single infection have been demonstrated to serve a multifunctional role; similar mechanism is being shown by the bovine hemoparasite, Babesia bovis (Allred 2001; Allred et al. 1994). At least two interrelated mechanisms have been proposed to establish persistent infections in cattle: cytoadhesion of infected erythrocytes containing mature parasites and rapid antigenic variation of the cytoadhesion ligands (Allred et al. 1994; O'Connor et al. 1999). The second mechanism is supposed to protect the cytoadhesion from ongoing and adaptive immune responses (Allred et al. 1994). The variant erythrocyte surface antigen-1
(VESA1), which is a size-polymorphic heterodimeric protein composed of two subunits $1 \mathrm{a}$ and $1 \mathrm{~b}$, is responsible for mediation of antigenic variation and cytoadhesion (Allred et al. 2000, 1993). The vesl $\alpha$ and vesl $\beta$ genes, from clearly related but structurally distinct branches of the ves gene family, encode the two subunits VESA1a and 1b, respectively (Allred et al. 2000; Xiao et al. 2010). The ves gene family is conserved among different Babesia species (B. bigemina, B. divergens, $B$. ovata, and B. bovis) but greatly diversified in protein structure and gene repertoire, involving persistent challenges of host-parasite interactions for millions of years. B. bovis VESA1 proteins possess cysteine-rich CKRD and VDCS domains, which are lacking in VESA1 proteins in B. divergens, B. bigemina, and B. ovata (Jackson et al. 2014).

This study is the first to identify and characterize VESA1 protein of $B$. orientalis (BoVESA1) with molecular weight $\sim 28 \mathrm{kDa}$. The immunogenicity and localization of BoVESA1 were identified by western blotting and immunofluorescent assay (IFA). The results indicate that BoVESA1 is a promising antigen for diagnostic usage and vaccine development against $B$. orientalis; the results are constructive to ensure the promotion of relevant research.

\section{Materials and methods}

\section{Cloning and sequencing of the BoVESA1 gene}

The genome of $B$. orientalis (unpublished data) was screened for the reported VESA1 amino acid sequences of B. bovis (T2Bo) (GenBank: XP_001609533.1) using TBLASTN. The gene with a remarkable similarity was designated as the BoVESA1 gene. Complete recombinant plasmids were constructed using the homologous recombination method. Specific primers for the BoVESA1 gene and pGEX-6p-1 vector (Table 1) were designed using Clone Manager software and then synthesized (Tianyi Huiyuan Biological Technology, Wuhan, China).

The BoVESA1 gene was amplified from both $B$. orientalis cDNA and gDNA obtained from previous studies (Guo et al. 2018a) with Phanta Max Super-Fidelity DNA Polymerase (Vazyme, Nanjing, China). The thermal cycling
Table 1 Primers used for construction of pGEX-6p1-BoVESA1 recombinant plasmids in this study

\begin{tabular}{lll}
\hline Primers & Primer sequences $\left(5^{\prime}-3^{\prime}\right)$ & Restriction enzyme \\
\hline BoVESA1-F & CCCTGGGATCCCCGGAATTCATGTCCTGT & BamHI \\
& CAAACGGTG & \\
BoVESA1-R & AGTCACGATGCGGCCGCTTGGACCACCTT & NotI \\
& GACCAG & NotI \\
pGEX-6p-1-vector-F & AGCGGCCGCATCGTGACT & BamHI \\
pGEX-6p-1-vector-R & GAATTCCGGGGATCCCAGGG & \\
\hline
\end{tabular}


parameters were as follows: activation at $95^{\circ} \mathrm{C}$ for $3 \mathrm{~min} ; 35$ cycles of denaturation at $95{ }^{\circ} \mathrm{C}$ for $15 \mathrm{~s}$, annealing at $54{ }^{\circ} \mathrm{C}$ for $15 \mathrm{~s}$, extension at $72{ }^{\circ} \mathrm{C}$ for $1 \mathrm{~min}$, and a final extension at $72{ }^{\circ} \mathrm{C}$ for $5 \mathrm{~min}$. The vector pGEX-6p-1 plasmid DNA was linearized for homologous recombination. The thermal cycling parameters were as follows: initial denaturation at $95^{\circ} \mathrm{C}$ for $3 \mathrm{~min} ; 35$ cycles of denaturation at $95^{\circ} \mathrm{C}$ for $15 \mathrm{~s}$, annealing at $61{ }^{\circ} \mathrm{C}$ for $15 \mathrm{~s}$, extension at $72{ }^{\circ} \mathrm{C}$ for $4 \mathrm{~min}$, and a final extension at $72{ }^{\circ} \mathrm{C}$ for $5 \mathrm{~min}$.

The PCR products were electrophoresed using 1.5\% ethidium bromide-stained agarose gel and purified using a DNA Gel Extraction Kit (TSINGKE Biological Technology, Beijing, China). The purified BoVESA1 gene was ligated into the extracted product of the pGEX-6p-1 vector. All the constructs were confirmed by DNA sequencing (Tianyi Huiyuan Biological Technology, Wuhan, China).

\section{Phylogenetic and bioinformatics analysis}

To attain a holistic comprehension of the evolutionary genetics analysis of the BoVESA1 gene, the amino acid sequence of BoVESA1 was aligned with VESA1 sequences in the database by BLASTP. Phylogenetic analysis was performed by the neighbor-joining method, owing to its superiority to classical programs based on multiple alignments, using MEGA7 software (Kumar et al. 2016). The amino acid (aa) sequence of BoVESA1 was analyzed using the ExPASY online tool (http://www.expasy.org/translate/). Open reading frames (ORFs) were searched using ORF finder (https:// www.ncbi.nlm.nih.gov/orf finder/). The putative signal peptide and potential subsequence motifs were predicted using SignalP 5.0 Server (http://www.cbs.dtu.dk/services/Signa 1P/) and Motif Scan Server (https://myhits.isb-sib.ch/cgibin/motif_scan/). The grand average of hydropathicity index (GRAVY) of BoVESA1 was calculated using the ExPASY ProtParam tool (https://web.expasy.org/protparam/). Subsequently, protein sequences were analyzed in detail by predicting the putative glycosylphosphatidylinositol (GPI) anchors with GPI Prediction Server 3.0 (http://mendel.imp. ac.at/sat/gpi/gpi_server.html) and predicting the transmembrane (TM) regions using https://embnet.vital-it.ch/softw are/TMPRED_form.html\#opennewwindow. The secondary structure of BoVESA1, surface probability, hydrophilicity, flexible region, and antigenic index were analyzed and predicted by the PROTEAN program of the Lasergene ${ }^{\circledR}$ software package in DNAstar.

The complete BoVESA1 amino acid sequence was submitted to the I-TASSER server (https://zhanglab.ccmb.med. umich.edu/) for predicting its three-dimensional structure. There were approximately three sessions to predict the three-dimensional structure of BoVESA1. First, I-TASSER modeling started from the structure templates identified by the local meta-threading server (LOMETS) from the
PDB library. The 10 best threading templates with higher structure accuracy of the conserved regions, owing to their highest significance in the threading alignments and hence a higher normalized Z-score, were selected from each threading program (Yang and Zhang 2015; Roy et al. 2012). Second, based on the significance of threading template alignments and the convergence parameters of the structure assembly simulations, five models corresponding to the five largest structure clusters were recorded using the SPICKER program (Yang et al. 2015; Zhang 2009). The model with the best quality among five selected models was regarded as the preliminary tertiary structure of BoVESA1 for prediction. Eventually, TM-align, a structural alignment program, was performed to match the first I-TASSER model to all structures in the PDB library, and the top 10 proteins were reported to have the closest structural similarity.

\section{Expression and purification of the recombinant proteins}

The recombinant plasmid (pGEX-6p-1-BoVESA1) was transformed into E. coli BL21 (DE3) strain. Smallscale culture was performed under isopropyl- $\beta$-Dthiogalactopyranoside (IPTG) at concentration of $1 \mathrm{mM}$ and the induction was continued at $37^{\circ} \mathrm{C}$ for $5 \mathrm{~h}$ to have the capacity and form of proteins certified by gel electrophoresis. BoVESA1 was expressed as a glutathione-S-transferase (GST)-fusion protein in E. coli as an inclusion body. The transformed E. coli were then lysed by ultrasonication in PBS $(\mathrm{pH}=7.4)$ containing phenylmethylsulfonyl fluoride (PMSF) and centrifuged further at $12,000 \times g$ for $30 \mathrm{~min}$ at $4{ }^{\circ} \mathrm{C}$. Precipitates were treated with PBS containing $0.2 \mathrm{~g} /$ $\mathrm{mL}$ sodium $\mathrm{N}$-dodecanoyl sarcosinate (SKL) for $2 \mathrm{~h}$ at $25^{\circ} \mathrm{C}$ and then centrifuged at $15,000 \times g$ for $10 \mathrm{~min}$ at $4{ }^{\circ} \mathrm{C}$. Supernatant containing rBoVESA 1 was treated with a refolding buffer $(0.2 \mathrm{~g} / \mathrm{mL}$ PEG-4000, $1 \mathrm{mM}$ oxidized glutathione, and $2 \mathrm{mM}$ reduced glutathione) overnight at $4{ }^{\circ} \mathrm{C}$ to allow complete refolding of the protein and thus to obtain purified protein.

\section{Development of polyclonal antibodies}

Kunming mice (specific pathogen-free) were subcutaneously injected with $100 \mu \mathrm{g}$ of purified rBoVESA1 emulsified in Freund's complete adjuvant (FCA, Sigma, San Francisco, CA, USA). Mice were injected with the same antigen (concentrated dose) in Freund's incomplete adjuvant (FIA, Sigma) on days 14,28 , and 42 after the first immunization. Serum samples from immunized mice and one healthy mouse were collected 14 days after the last immunization. The collected samples were stored at $-20{ }^{\circ} \mathrm{C}$ for further use. 


\section{SDS-PAGE and western blot analysis}

rBoVESA 1 was subjected to $12 \%$ sodium dodecyl-sulfate polyacrylamide gel electrophoresis (SDS-PAGE) and transferred to a nitrocellulose membrane (Merck Millipore, Kenny, NJ, USA), which was subsequently blocked with $5 \%(\mathrm{w} / \mathrm{v})$ skimmed milk in Tris-buffered saline Tween-20 (TBST). The membranes probed with serum (1:50 dilution) from the buffalo infected with $B$. orientalis or healthy water buffalo were incubated at $25^{\circ} \mathrm{C}$ for $1 \mathrm{~h}$ with goat anti-bovine IgG/HRP (1:2000) as secondary antibodies (Bioss, Beijing, China) and visualized using the electrochemiluminescence (ECL) method.

To determine the native BoVESA1 in merozoites of $B$. orientalis, proteins from the lysates of $B$. orientalis-infected buffalo erythrocytes and uninfected buffalo erythrocytes were first electrophoresed through 12\% SDS-PAGE gel, transferred to a nitrocellulose membrane, and probed with anti-rBoVESA1 mice serum (1:50 dilution) or the naive mouse serum at the same dilution as controls. After three washes with Tris-buffered saline Tween-20 (TBST), the membranes were incubated with goat anti-mouse IgG/HRP (1:4000) as secondary antibodies (Bioss) and finally detected using the ECL approach.

\section{Indirect immunofluorescence assay}

To determine the cellular localization of BoVESA1, water buffalo RBCs infected with $B$. orientalis at merozoite stages (4\% parasitemia) were smeared on glass slides, air-dried, and then fixed in cold acetone: methanol (50\% v/v) solution for $20 \mathrm{~min}$ at $-20^{\circ} \mathrm{C}$. After three washes with cold $1 \times \mathrm{PBS}$, smears of blood cells were permeabilized with $0.1 \%$ Triton $\mathrm{X}-100$ and blocked with $1 \%$ BSA and then incubated with the anti-rBoVESA1 mouse serum (1:100) and the naive mouse serum $\left(1: 100\right.$, negative control) for $1 \mathrm{~h}$ at $37^{\circ} \mathrm{C}$. The secondary antibody was goat anti-mouse IgG (Alexa Fluor 594) diluted 1000 times with $1 \times \mathrm{PBS}$, followed by parasite nucleus staining with Hoechst stain for $1 \mathrm{~h}$ at $37^{\circ} \mathrm{C}$. Finally, $10 \mu \mathrm{L}$ of anti-fluorescence quenching agent was used to prevent the fluorescence quenching effect, and the images were obtained using a fluorescence microscope.

\section{Results}

\section{Characterizations of BoVESA1 gene}

The BoVESA1 gene contained a 753-bp open reading frame without introns and encoded a polypeptide of 250 aa (Fig. 1). The nucleotide sequence was submitted to GenBank with the accession number MT721826. In addition, the BoVESA1 gene was expressed as a GST-fusion protein with a predicted

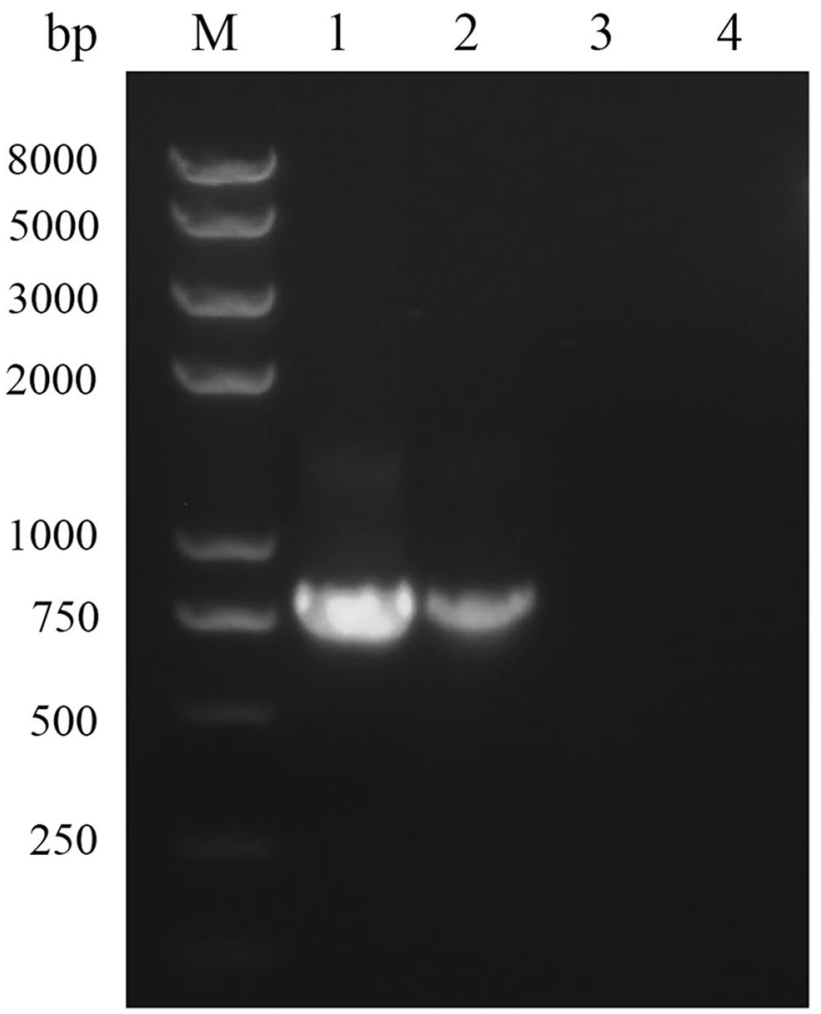

Fig. 1 PCR amplification of BoVESA1 gene from $B$. orientalis gDNA and cDNA. Lane M: marker; lane 1: amplicon from gDNA; lane 2: amplicon from $B$. orientalis cDNA; lane 3: amplicon from distilled water as blank control; lane 4: amplicon from uninfected buffalo erythrocytes cDNA as negative control

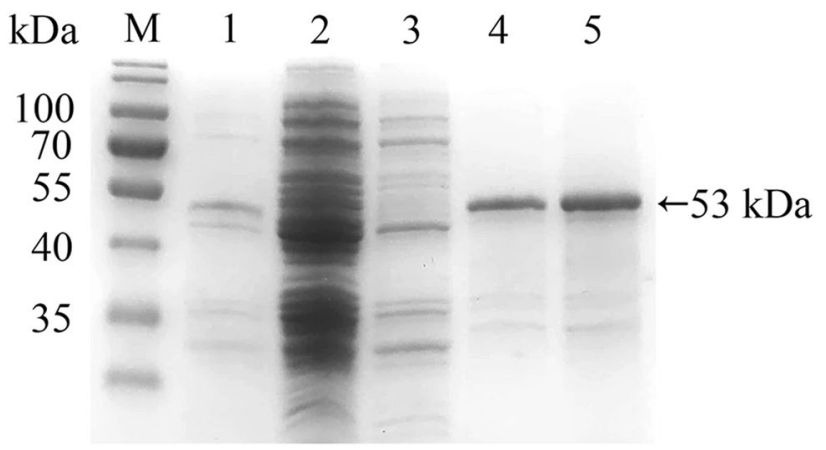

Fig. 2 SDS-PAGE expression and purification of rBoVESA1. Lane M: molecular weight marker; lane 1: induced pGEX-6p-1-BoVESA1; lane 2: non-induced pGEX-6p-1-BoVESA1 as control; lane 3: supernatant of pGEX-6p-1-BoVESA1; lane 4: pellet of pGEX-6p-1BoVESA1; lane 5: purified rBoVESA1. The corresponding bands are indicated by arrows

molecular weight of $53 \mathrm{kDa}$ in the SDS-PAGE. It was also demonstrated that the expressed proteins were insoluble and existed in the form of inclusion bodies (Fig. 2). Primary purified rBoVESA1 was obtained, collected, and dialyzed to immunize mice. 


\section{Phylogenetic and bioinformatics analysis}

The amino acid sequence of BoVESA1 was aligned with VESA1 sequences in the database. Among all the amino acid sequences producing significant alignments, BoVESA1 was most closely related to VESA1 protein in B. bovis (XP_001611438.1) with an identity of 34\% and a query cover of $92 \%$. Additionally, BoVESA1 showed $30 \%$ and $21 \%$ identity to VESA1 of Babesia sp. Xinjiang (XP_028870229.1) and B. bigemina (XP_012769198.1), respectively. The evolutionary relationships between VESA1 amino acid sequences from Babesia bovis, Babesia orientalis, Babesia bigemina, Babesia ovata, Babesia microti, and Babesia sp. Xinjiang were analyzed using the neighbor-joining method in the MEGA7 software, using $P$. falciparum erythrocyte membrane protein 1 (PfEMP-1) as an outlier (Fig. 3). According to the phylogenetic analysis, the results showed that BoVESA1 has a closer relationship with VESA1 of B. bovis than those of other Babesia species.

Four important indexes, including hydrophilicity, flexible regions, antigenic index, and surface probability, were predicted on the basis of some relevant algorithms that were inclusive of Kyte-Doolittle, Karplus Dchulz, Jameson-Wolf, and Emini, through the PROTEAN program of the Lasergene ${ }^{\circledR}$ software package in DNAstar. Hydrophilic regions were predicted to take up more of the amino acid sequence than hydrophobic regions; the grand average of hydropathicity (GRAVY) index of BoVESA1 was -0.284 , implying a high hydrophilicity of BoVESA1. The structures of turn and coil had certain flexibility and deformability, which may promote the binding process with antibodies and serve as a possible epitope, in contrast to the structures of $\alpha$-helices and $\beta$-sheets, which are indeformable and uneasy to bind antibodies. Regions with high accessibility are distributed on the surface of the antigen to bind with antibodies more easily. High antigenic regions with a uniform distribution throughout the amino acid sequence focused mainly in the range of 7 to 62 and 112 to 163 aa. These four related indices are needed to evaluate potential B cell epitopes: good hydrophilicity and antigenicity as well as high accessibility and flexibility. Conclusively, three regions of 9 to 13 aa, 51 to 62 aa,
Fig. 3 Molecular phylogenetic analysis of BoVESA1 amino acid sequences by the neighborjoining method. The tree is drawn to scale, with branch lengths measured in the number of substitutions per site. The analysis involved 24 amino acid sequences. All positions containing gaps and missing data were eliminated. There were a total of 172 positions in the final dataset. The sequence newly identified in this study is presented with a bold font. GenBank accession numbers are indicated

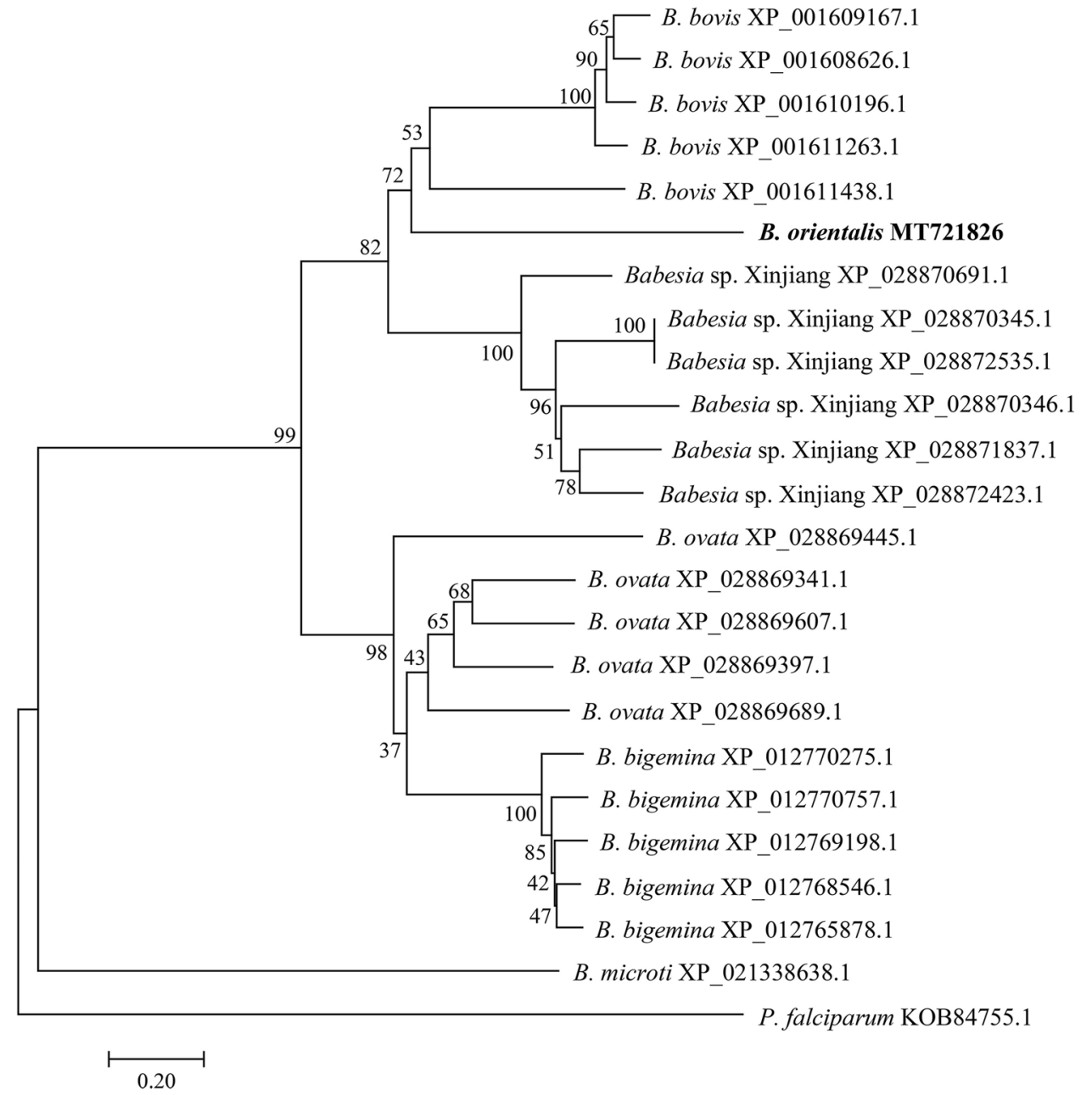


and 147 to 152 aa are most likely to be potential B cell epitopes.

The BoVESA1 gene was predicted to have one exon and no intron with a lack of GPI anchor sites and N-terminal signal peptide. Consistent with the VESA1 proteins of other Babesia species, BoVESA1 was revealed to contain a transmembrane region (59-84 aa). The motif scanning result of the amino acid sequence of BoVESA1 was obtained from Motif Scan Server. It mainly consisted of an N-glycosylation site between 10 and 13 aa, four casein kinase II phosphorylation sites in the range of 5 to 238 aa, three protein kinase $\mathrm{C}$ phosphorylation sites in the range of 56 to 146 aa, seven $\mathrm{N}$-myristoylation sites in the range of 35 to 245 aa, and one RGD cell attachment sequence (146-148 aa).

The amino acid sequence of BoVESA1 was processed using the I-TASSER software to predict the three-dimensional structure. A schematic illustration of this process is shown in Fig. 4. Among the proteins selected from the PDB library, a light-harvesting chlorophyll a/b-binding (LHC) protein (PDB code: 6ijj3) possessed the closest structural similarity to the BoVESA1 model, which is encoded by the lhca3 gene of Chlamydomonas reinhardtii $(\mathrm{Cr})$; LHC being a membrane protein can collect and transfer light energy to the reaction centers of the photosystem I (PSI) (Su et al. 2019). Because of its similar structure to 6ijj3, BoVESA1 was speculated to be combined with some functional proteins to transmit energy and signal, which remains to be deeply studied in further research.

\section{Identification of the recombinant and native BoVESA1}

To evaluate the immunoreactivity of BoVESA1, rBoVESA1 was reacted with the serum collected from $B$. orientalis-infected and uninfected buffalo and analyzed by western blotting. Only one specific band corresponding to rBoVESA1 (53 $\mathrm{kDa})$ appeared in the serum from the infected water buffalo (Fig. 5a), but no band was observed in the serum of the control buffalo. In addition, BoVESA1 has been predicted to have good hydrophilicity and antigenicity; thus, BoVESA1 may be conceived as a good diagnostic antigen for the detection of $B$. orientalis.

The identification of native BoVESA1 was also performed by incubating the lysates of $B$. orientalis-infected erythrocytes with anti-rBoVESA1 serum. The results displayed a $\sim 28 \mathrm{kDa}$ band, which was similar to the anticipated size of native BoVESA1 (Fig. 5b), while the control group showed no band. The presence of a $\sim 28 \mathrm{kDa}$ band demonstrated the excellent immunological properties of rBoVESA 1 and confirmed the presence of native BoVESA1 in $B$. orientalis merozoite.

\section{Localization of BoVESA1}

IFA was performed to determine the localization of BoVESA1 by mouse anti-rBoVESA1 polyclonal antibodies serum and naive mouse serum. The results showed a strong signal in infected RBCs (Fig. 6), while no fluorescence was detected in the negative control.

\section{Discussion}

Several studies have been conducted on B. orientalis including analyzing and sequencing the mitochondrial and apicoplast genomes of B. orientalis (He et al. 2017, 2014; Huang et al. 2015). However, it still remains unclear about the mechanism of invasion of parasites into host erythrocytes and avoiding host immune responses.
Fig. 4 Schematic illustrations of predicted tertiary structures of BoVESA1. a Predicted tertiary structure of BoVESA1 showing $\alpha$-helices (red) and coils (green). The tertiary structure contains nine $\alpha$-helices $(\alpha 1$ and $\alpha 9)$. b Tertiary structure of light-harvesting chlorophyll a/b-binding (LHC) protein (PDB code: 6ijj3). The tertiary structure in identical orientations contains eight $\alpha$-helices ( $\alpha 1$ and $\alpha 8$ )

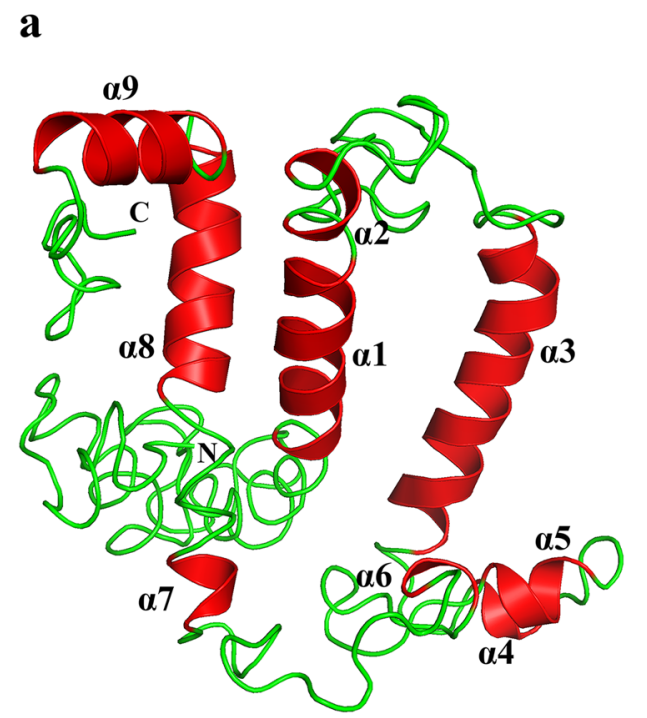

b

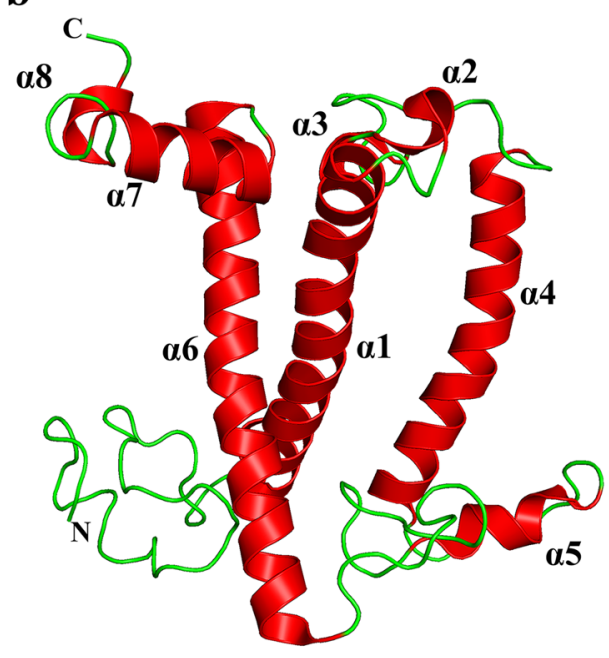


a

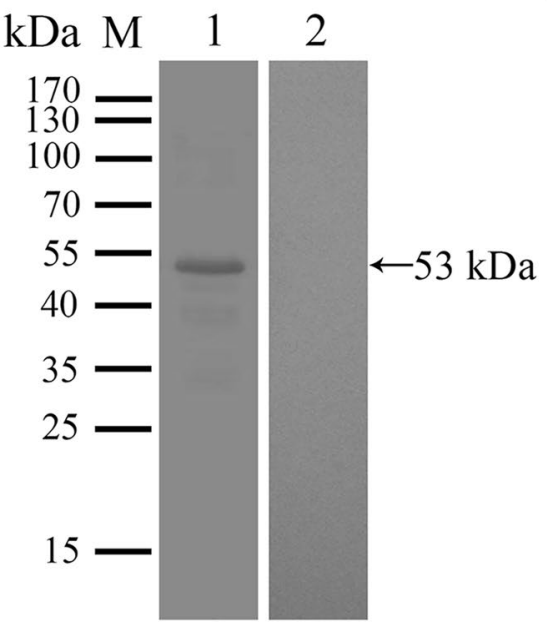

b

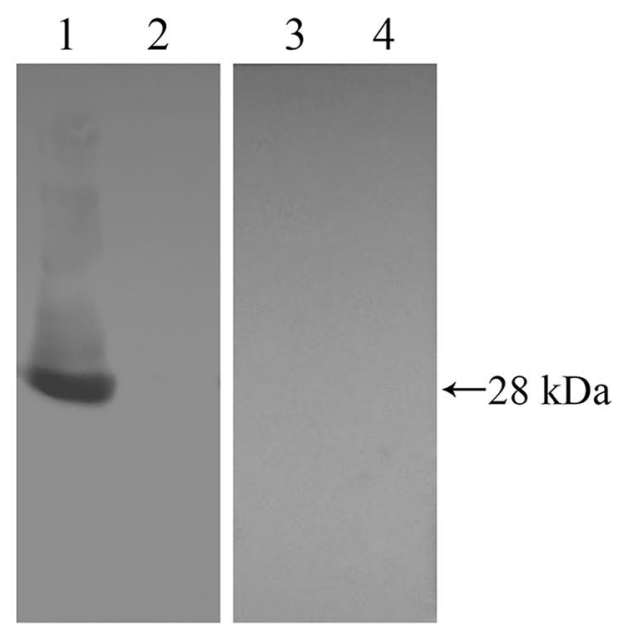

Fig. 5 Western blot identification of immunoreactivity and native form of BoVESA1. a Lane M: molecular weight marker; lane 1: rBoVESA1 reacted with $B$. orientalis positive serum; lane 2: rBoVESA1 probed with the serum of uninfected buffalo. $\mathbf{b}$ Identification of the native form of BoVESA1 in B. orientalis merozoite lysate. Lane 1: reaction of lysate of $B$. orientalis-infected buffalo erythro- cytes with the serum against rBoVESA1; lane 2: reaction of lysate of uninfected buffalo erythrocytes with the serum against rBoVESA1; lane 3: lysate of $B$. orientalis-infected buffalo erythrocytes probed with the serum of naïve mice; lane 4: lysate of uninfected buffalo erythrocytes probed with the serum of naïve mice. The corresponding bands are indicated by arrows
Fig. 6 Localization of BoVESA1. Immunofluorescence and electron microscopy analysis of BoVESA1 in the smears. PcAb-BoVESA1 (red) and nucleus staining of Hoechst (blue). a Reactivity of the antirBoVESA1 serum with intracellular parasite. b Negative control: the primary antibody was serum from a naïve mouse. Scale bars: $1 \mu \mathrm{m}$

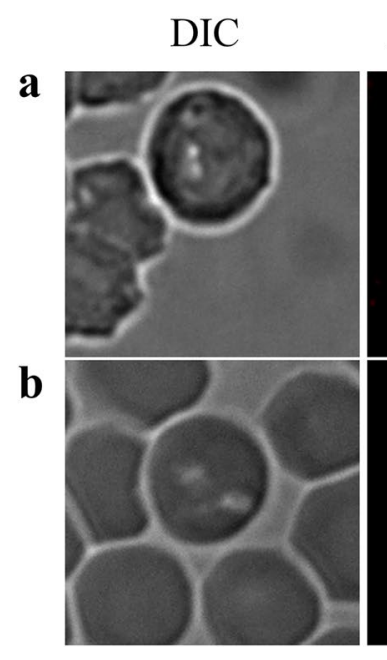

Anti-BoVESA1

Hoechst

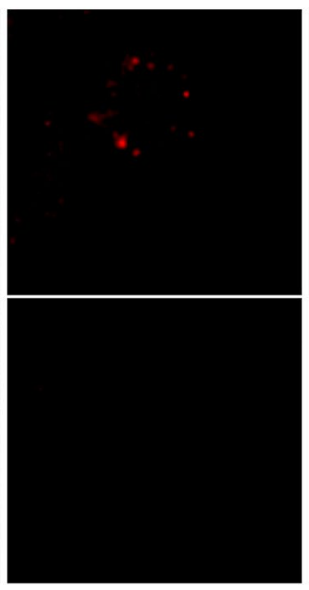

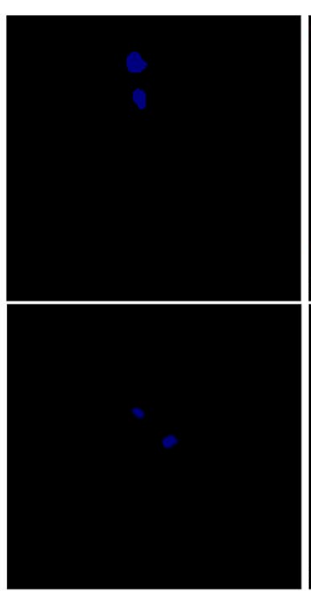

Merge

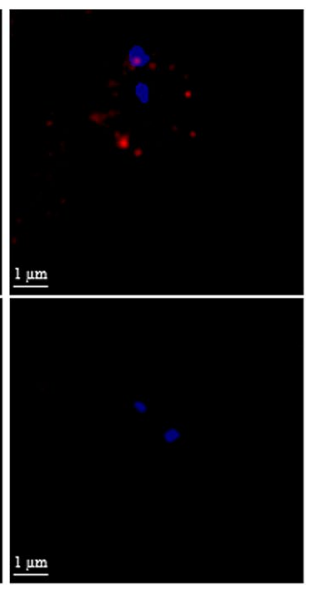

Persistent infections in $B$. bovis could be established with the involvement of at least two mechanisms including endothelial cytoadhesion and antigenic variation. Sequestration of infected erythrocytes (David et al. 1983; Wright 1972) results from cytoadhesion of the infected red blood cells to the capillary along the post-capillary venous endothelium via some knob-like structures emerging from its surface (Aikawa et al. 1985; Hutchings et al. 2007; O'Connor and Allred 2000; O'Connor et al. 1999; Schmidt et al. 1982). For antigenic variation, it was proposed to make sure adhesion function escaped from adaptive host immune responses (Biggs et al. 1992; Leech et al. 1984; Magowan et al. 1988; O'Connor et al. 1997). Both of these functions are related to the VESA1 on the surface of infected erythrocytes (Allred et al. 2000, 1994; O'Connor and Allred 2000; O'Connor et al. 1997). Usually, it exists in two different types, VESAla and VESA1b, encoded by ves $1 \alpha$ and ves $1 \beta$, respectively; a small third branch of only three copies, ves $1 \gamma$, has been observed as well but is of unknown function (Allred 2019). The transcription of B. bovis ves gene occurs at a "locus for active transcription" (LAT) (Al-Khedery and Allred 2006), including ves $1 \alpha / v e s 1 \beta$ or ves $1 \alpha / v e s 1 \alpha$ gene pairs with divergent orientations. The pathogens such as Plasmodium falciparum, African trypanosomes, and Candida albicans have the ability to escape immune responses due to continuous generation of variant antigens. The ves 
genes in Babesia spp. also follow similar mechanism as the var genes in the malaria parasite Plasmodium falciparum (Bopp et al. 2013) and the vsg genes in Trypanosoma brucei (Jackson et al. 2012).

The ves gene family is relatively conserved for Babesia spp. but highly diversified in protein structure and gene repertoire, owing to gene duplication and expansion over the years. The ves $1 \alpha /$ ves $1 \beta$ branches in $B$. bovis do not show significant orthologous relationships with the vesla/ves $1 b$ branches in B. bigemina or B. ovata (Jackson et al. 2014). Moreover, cysteine-rich CKRD and VDCS domains of $B$. bovis are lacking from VESA1 proteins encoded by vesla/ves $1 b$ genes in B. bigemina and B. ovata (Jackson et al. 2014; Yamagishi et al. 2017), which may be responsible for the lack of cytoadhesion and knob-like structures in these species. Furthermore, ves-like short genes appeared after rapid divergence of ves 1 structures (Jackson et al. 2014), implying its specific function and evolutionary dynamics.

Although plenty of studies have been conducted on the identification of VESA1 proteins in Babesia spp., but none of them focused on B. orientalis. In the present study, the VESA1 protein of $B$. orientalis was identified and characterized for the first time. We cloned and sequenced an intronless VESA1 gene with an open reading frame of $753 \mathrm{bp}$ in $B$. orientalis. Aiming to obtain a detailed understanding of the genetic relationships among different species, the VESA1 amino acid sequences were aligned to each other in BLAST program. The amino acid homology of BoVESA1 to VESA1 protein of B. bovis and Babesia sp. Xinjiang was $34 \%$ and $30 \%$, respectively, suggesting that BoVESA1 has a closer relationship with B. bovis. SBP3, SBP4, RON5, and thrombospondin-related anonymous protein 2 (TRAP2) amino acid sequences of $B$. orientalis have also been reported closer to those of B. bovis (Guo et al. 2018a, b; Tian et al. 2020; Zhan et al. 2019). BoVESA1 was also predicted to have one RGD cell attachment region (146-148 aa), which was possible to facilitate potential cytoadhesion and transmit signals. Moreover, a $\sim 28 \mathrm{kDa}$ band was detected in the lysates of $B$. orientalis-infected buffalo erythrocytes reacted with mouse anti-rBoVESA1 serum, indicating the existence of native VESA1 in B. orientalis merozoite. The IFA results showed a strong fluorescence signal of BoVESA1 (captured in the intracellular stage) in infected RBCs using anti-rBoVESA1 serum; however, the secretion of BoVESA1 into host RBCs might be related to the presence of a transmembrane region close to the $\mathrm{N}$-terminal. Furthermore, in B. bovis, VESA1 protein was confirmed to be localized on the surface of infected erythrocytes, but the mechanism by which VESA1 proteins were trafficked to erythrocyte membranes remains to be further investigated. So far, researchers have discovered that overexpression of spherical body protein (SBP2t11) can cause reduced cytoadhesion of infected erythrocytes (Gallego-Lopez et al. 2019). Since VESA1 in
B. bovis was mainly responsible for cytoadhesion, upregulation of the SBP2t11 protein may impede the export of VESA1 proteins to the surface of infected erythrocytes, implying the spherical body compartment was possible to be involved in the export pathway (Allred 2019). Despite vital importance of the invasion stage of parasites, deeper investigations on the post-invasion stage are still necessary because there can be far longer time of being exposed to the host immune system during post-invasion stage than invasion stage and may cause potential risks to animals.

\section{Conclusion}

In this study, BoVESA1 was cloned, sequenced, and expressed as a $53 \mathrm{kDa}$ GST-fusion protein with the native form being identified as $\sim 28 \mathrm{kDa}$ by western blot and confirmed to have excellent immunogenicity that indicates that BoVESA1 can serve as a good candidate diagnostic antigen for the detection of $B$. orientalis infection. The IFA results showed a strong signal in infected RBCs. The identification of VESA1 in B. orientalis in the current study may facilitate further investigation on the ves gene and boost the development of effective therapy for $B$. orientalis infection.

Author contribution $\mathrm{ZH}, \mathrm{LH}$, and JZ designed the study and drafted the manuscript. $\mathrm{ZH}$ and $\mathrm{YZ}$ performed the experiments. $\mathrm{ZN}$ and $\mathrm{XS}$ participated in the data analysis. All authors read and approved the final manuscript.

Funding This study was supported by the National Natural Science Foundation of China (Grant No. 31930108 and 31772729), the Natural Science Foundation of Hubei Province (Grant No. 2017CFA020), and the Fundamental Research Funds for the Central Universities in China (Project 2662020DKPY016).

Data availability The nucleotide sequence was annotated and submitted to the NCBI GenBank under the accession number MT721826.

\section{Declarations}

Ethics statement The experimental animals were housed and treated in accordance with the stipulated rules for the regulation of the administration of affair concerning experimental animals of People's Republic of China. All experiments were performed with the approval of Laboratory Animals Research Centre of Hubei Province and the Ethics Committee of Huazhong Agricultural University (Permit number: HZAUMO-2019-016).

Conflict of interest The authors declare no competing interests.

\section{References}

Aikawa M, Rabbege J, Uni S, Ristic M, Miller LH (1985) Structural alteration of the membrane of erythrocytes infected with Babesia 
bovis. Am J Trop Med Hyg 34:45-49. https://doi.org/10.4269/ ajtmh.1985.34.45

Al-Khedery B, Allred DR (2006) Antigenic variation in Babesia bovis occurs through segmental gene conversion of the ves multigene family, within a bidirectional locus of active transcription. Mol Microbiol 59:402-414. https://doi.org/10.1111/j.1365-2958.2005. 04993.x

Allred DR (2001) Antigenic variation in babesiosis: is there more than one 'why'? Microbes Infect 3:481-491. https://doi.org/10.1016/ s1286-4579(01)01404-6

Allred DR (2019) Variable and variant protein multigene families in Babesia bovis persistence. Pathogens 8.https://doi.org/10.3390/ pathogens 8020076

Allred DR, Hines SA, Ahrens KP (1993) Isolate-specific parasite antigens of the Babesia bovis-infected erythrocyte surface. Mol Biochem Parasitol 60:121-132. https://doi.org/10.1016/01666851(93)90035-v

Allred DR, Cinque RM, Lane TJ, Ahrens KP (1994) Antigenic variation of parasite-derived antigens on the surface of Babesia bovisinfected erythrocytes. Infect Immun 62:91-98

Allred DR et al (2000) The ves multigene family of B. bovis encodes components of rapid antigenic variation at the infected erythrocyte surface. Mol Cell 5:153-162. https://doi.org/10.1016/s10972765(00)80411-6

Arnarson H, Palsson A, Gudnadottir M, Andresdottir V (2017) Maedivisna virus persistence: antigenic variation and latency. Comp Immunol Microbiol Infect Dis 55:6-12. https://doi.org/10.1016/j. cimid.2017.08.003

Berdyev A (1985) Evolution of the basic types of life cycle in ixodid ticks (Ixodidae). Parazitologiia 19:134-138

Biggs BA, Anders RF, Dillon HE, Davern KM, Martin M, Petersen C, Brown GV (1992) Adherence of infected erythrocytes to venular endothelium selects for antigenic variants of Plasmodium falciparum. J Immunol 149:2047-2054

Biswal JK et al (2019) Genetic and antigenic variation of footand-mouth disease virus during persistent infection in naturally infected cattle and Asian buffalo in India. PLoS ONE 14:e0214832. https://doi.org/10.1371/journal.pone.0214832

Bopp SER et al (2013) Mitotic evolution of Plasmodium falciparum shows a stable core genome but recombination in antigen families. Plos Genet 9:e1003293. https://doi.org/10.1371/journal.pgen. 1003293

David PH, Hommel M, Miller LH, Udeinya IJ, Oligino LD (1983) Parasite sequestration in Plasmodium falciparum malaria: spleen and antibody modulation of cytoadherence of infected erythrocytes. Proc Natl Acad Sci U S A 80:5075-5079. https://doi.org/ 10.1073/pnas.80.16.5075

Deitsch KW, Moxon ER, Wellems TE (1997) Shared themes of antigenic variation and virulence in bacterial, protozoal, and fungal infections. Microbiol Mol Biol Rev 61:281-293

Gallego-Lopez GM et al (2019) Up-regulated expression of spherical body protein 2 truncated copy 11 in Babesia bovis is associated with reduced cytoadhesion to vascular endothelial cells. Int $\mathbf{J}$ Parasitol 49:127-137. https://doi.org/10.1016/j.ijpara.2018.05.015

Guo J et al (2018a) A novel Babesia orientalis 135-kilodalton spherical body protein like: identification of its secretion into cytoplasm of infected erythrocytes. Parasit Vectors 11:205. https://doi.org/10. 1186/s13071-018-2795-7

Guo J et al (2018b) Characterization of a novel secretory spherical body protein in Babesia orientalis and Babesia orientalis-infected erythrocytes. Parasit Vectors 11:433. https://doi.org/10.1186/ s13071-018-3018-y

$\mathrm{He}$ L et al (2014) Mitochondrial genome of Babesia orientalis, apicomplexan parasite of water buffalo (Bubalus babalis, Linnaeus, 1758) endemic in China. Parasit Vectors 7:82. https://doi.org/10. $1186 / 1756-3305-7-82$
He L, Liu Q, Yao B, Zhou Y, Hu M, Fang R, Zhao J (2017) A historical overview of research on Babesia orientalis, a protozoan parasite infecting water buffalo. Front Microbiol 8:1323. https:// doi.org/10.3389/fmicb.2017.01323

Huang Y et al (2015) Characterization and annotation of Babesia orientalis apicoplast genome. Parasit Vectors 8:543. https://doi. org/10.1186/s13071-015-1158-x

Hutchings CL et al (2007) New insights into the altered adhesive and mechanical properties of red blood cells parasitized by Babesia bovis. Mol Microbiol 65:1092-1105. https://doi.org/10.1111/j. 1365-2958.2007.05850.x

Jackson AP et al (2012) Antigenic diversity is generated by distinct evolutionary mechanisms in African trypanosome species. PNAS 109:3416-3421. https://doi.org/10.1073/pnas.11173 13109

Jackson AP et al (2014) The evolutionary dynamics of variant antigen genes in Babesia reveal a history of genomic innovation underlying host-parasite interaction. Nucleic Acids Res 42:7113-7131. https://doi.org/10.1093/nar/gku322

Kivaria FM (2006) Estimated direct economic costs associated with tick-borne diseases on cattle in Tanzania. Trop Anim Health Prod 38:291-299. https://doi.org/10.1007/s11250-006-4181-2

Klompen JS, Black WCT, Keirans JE, Oliver JH Jr (1996) Evolution of ticks. Annu Rev Entomol 41:141-161. https://doi.org/10.1146/ annurev.en.41.010196.001041

Kumar S, Stecher G, Tamura K (2016) MEGA7: molecular evolutionary genetics analysis version 7.0 for bigger datasets. Mol Biol Evol 33:1870-1874. https://doi.org/10.1093/molbev/msw054

Langsley G, Roth C (1987) Antigenic variation in parasitic protozoa. Microbiol Sci 4:280-285

Leech JH, Barnwell JW, Miller LH, Howard RJ (1984) Identification of a strain-specific malarial antigen exposed on the surface of Plasmodium falciparum-infected erythrocytes. J Exp Med 159:15671575. https://doi.org/10.1084/jem.159.6.1567

Magowan C, Wollish W, Anderson L, Leech J (1988) Cytoadherence by Plasmodium falciparum-infected erythrocytes is correlated with the expression of a family of variable proteins on infected erythrocytes. J Exp Med 168:1307-1320. https://doi.org/10.1084/ jem.168.4.1307

Novacco M, Hofmann-Lehmann R, Grimm F, Meli ML, Stirn M (2019) Fatal acute babesiosis associated with Babesia venatorum infection (Babesia sp. EU1) in a captive reindeer calf in Switzerland. Vet Parasitol Reg Stud Rep 18:100336. https://doi.org/10.1016/j. vprsr.2019.100336

O'Connor RM, Allred DR (2000) Selection of Babesia bovis-infected erythrocytes for adhesion to endothelial cells coselects for altered variant erythrocyte surface antigen isoforms. J Immunol 164:2037-2045. https://doi.org/10.4049/jimmunol.164.4.2037

O'Connor RM, Lane TJ, Stroup SE, Allred DR (1997) Characterization of a variant erythrocyte surface antigen (VESA1) expressed by Babesia bovis during antigenic variation. Mol Biochem Parasitol 89:259-270. https://doi.org/10.1016/s0166-6851(97)00125-4

O'Connor RM, Long JA, Allred DR (1999) Cytoadherence of Babesia bovis-infected erythrocytes to bovine brain capillary endothelial cells provides an in vitro model for sequestration. Infect Immun 67:3921-3928

Palmer GH, Bankhead T, Seifert HS (2016) Antigenic variation in bacterial pathogens. Microbiol Spectr 4.https://doi.org/10.1128/ microbiolspec.VMBF-0005-2015

Ristic M (1988) Babesiosis of domestic animals and man. CRC Press, Boca Raton

Roy A, Yang J, Zhang Y (2012) COFACTOR: an accurate comparative algorithm for structure-based protein function annotation. Nucleic Acids Res 40:W471-477. https://doi.org/10.1093/nar/gks372

Schmidt JA et al (1982) Plasmodium falciparum malaria. An amelanotic melanoma cell line bears receptors for the knob ligand 
on infected erythrocytes. J Clin Invest 70:379-386. https://doi.org/ $10.1172 /$ jci110627

Su X et al (2019) Antenna arrangement and energy transfer pathways of a green algal photosystem-I-LHCI supercomplex. Nat Plants 5:273-281. https://doi.org/10.1038/s41477-019-0380-5

Tian Y et al (2020) Identification and characterizations of a rhoptries neck protein 5 (BoRON5) in Babesia orientalis. Parasitol Int 77:102106. https://doi.org/10.1016/j.parint.2020.102106

Wright IG (1972) An electron microscopic study of intravascular agglutination in the cerebral cortex due to Babesia argentina infection. Int J Parasitol 2:209-215. https://doi.org/10.1016/00207519(72)90008-2

Wright IG, Goodger BV, Clark IA (1988) Immunopathophysiology of Babesia bovis and Plasmodium falciparum infections. Parasitol Today 4:214-218. https://doi.org/10.1016/0169-4758(88)90161-5

Xiao YP, Al-Khedery B, Allred DR (2010) The Babesia bovis VESA1 virulence factor subunit $1 \mathrm{~b}$ is encoded by the 1 beta branch of the ves multigene family. Mol Biochem Parasitol 171:81-88. https:// doi.org/10.1016/j.molbiopara.2010.03.001

Yamagishi J, Asada M, Hakimi H, Tanaka TQ, Sugimoto C, Kawazu SI (2017) Whole-genome assembly of Babesia ovata and comparative genomics between closely related pathogens. BMC Genomics 18:832. https://doi.org/10.1186/s12864-017-4230-4
Yang J, Zhang Y (2015) I-TASSER server: new development for protein structure and function predictions. Nucleic Acids Res 43:W174-181. https://doi.org/10.1093/nar/gkv342

Yang J, Yan R, Roy A, Xu D, Poisson J, Zhang Y (2015) The I-TASSER suite: protein structure and function prediction. Nat Methods 12:7-8. https://doi.org/10.1038/nmeth.3213

Young AS, Morzaria SP (1986) Biology of babesia. Parasitol Today 2:211-219. https://doi.org/10.1016/0169-4758(86)90084-0

Zhan X et al (2019) Identification of a novel thrombospondin-related anonymous protein (BoTRAP2) from Babesia orientalis. Parasit Vectors 12:200. https://doi.org/10.1186/s13071-019-3457-0

Zhang Y (2009) I-TASSER: fully automated protein structure prediction in CASP8. Proteins 77(Suppl 9):100-113. https://doi.org/10. 1002/prot.22588

Publisher's note Springer Nature remains neutral with regard to jurisdictional claims in published maps and institutional affiliations. 